\title{
LIPOSARCOMA DEDIFERENCIADO DEL CORDÓN ESPERMÁTICO: DEGENERACIÓN DE UN LIPOMA PREVIO RESECADO
}

\author{
E. PEYRÍ REY, A. URBAN RAMÓN, M. MARTÍNEZ FERNÁNDEZ, \\ B. SANMARTI DA SILVA
}

Servicio de Urología. Hospital Comarcal de la Selva. Blanes. Girona.

Actas Urol Esp. 27 (5): 383-386, 2003

\section{RESUMEN}

"LIPOSARCOMA DEDIFERENCIADO DEL CORDÓN ESPERMÁTICO: DEGENERACIÓN DE UN LIPOMA PREVIO RESECADO"

Los sarcomas del cordón espermático son tumores de rara presentación. El liposarcoma dediferenciado representa sólo un $10 \%$ de los sarcomas del cordón espermático. Estos suelen ser tumores de gran tamaño y con una histología caracterizada por ser un liposarcoma bien diferenciado con áreas de sarcoma de alto grado. El TAC y la ecografía nos informarán del volumen, localización, homogeneidad de la masa, así como de las posibles adenopatías pelvianas y retroperitoneales. Son útiles en el seguimiento después del tratamiento. Nosotros presentamos un liposarcoma dediferenciado, de cordón espermático, al que previamente, se le extirpó en tres ocasiones lipomas en el mismo cordón espermático. Creemos que se trata de una degeneración del lipoma previo resecado. El tratamiento es la orquiectomía radical por vía inguinal amplia, siendo la radioterapia y la quimioterapia de ayuda complementaria, con valor incierto. Suelen presentar recidivas locales frecuentes después de la cirugía, pudiendo presentar metástasis hematógenas y en nódulos pelvianos. Presentan una supervivencia a los 5 y 10 años, de un 75\% y 63\% respectivamente.

PALABRAS CLAVE: Liposarcoma dediferenciado. Cordón espermático. Degeneración lipoma.

\section{ABSTRACT \\ "DEDIFFERENTIATED LIPOSARCOMA OF SPERMATIC CORD: DEGENERATION LIPOMA RESECTION PREVIOUS"}

Spermatic cord sarcomas are rare tumours. Dedifferentiated liposarcoma accounts for only $10 \%$ of all spermatic cord sarcomas. These are usually large-sized tumours histologically characterised for being well-differentiated liposarcomas with some high grade sarcoma areas. Volume, location, mass homogeneity as well as presence of pelvic and retroperitoneal adenopathies are reported by CT and ultrasound techniques. These are useful for post-treatment follow-up. This paper presents one spermatic cord, dedifferentiated liposarcoma from which lipomas from the same spermatic cord had been previously removed in three occasions. We believe this is a degeneration of the earlier resected lipoma. Management is by extended inguinal radical orchiectomy. Value of adjuvant radio- and chemotherapy is uncertain. Post-surgery local relapses are common, and haematogenous and pelvic nodes metastasis likely. Survival at 5 and 10 years is $75 \%$ and $63 \%$ respectively. 
$\mathrm{L}$ os tumores paratesticulares representan el 7$10 \%$ de las masas intraescrotales. La mayoría (75-90\%) del total se originan en el cordón espermático, siendo casi todos benignos (70\%). Los sarcomas casi representan el 30\% restante ${ }^{1}$. Ballo en el 2001 realiza una revisión de 32 sarcomas del cordón espermático, encontrando: 12 histiocitomas fibroso maligno, 8 liposarcomas, 6 leiomiosarcomas, 6 otros subtipos, de los cuales 3 correspondían a liposarcoma dediferenciado ${ }^{2}$. Presentamos un caso de liposarcoma dediferenciado del cordón espermático, en un enfermo al que previamente se le había resecado un lipoma de cordón en el mismo lado en tres ocasiones. Nosotros realizamos la última hace 3 años, por lo que después de revisar de nuevo la histología creemos que puede ser un caso que confirmaría la teoría de degeneración maligna de un lipoma en liposarcoma ${ }^{3}$.

\section{CASO CLÍNICO}

Varón de 52 años, que refiere como antecedentes previos, intervenciones por recidiva de un lipoma de cordón espermático en el lado izquierdo, en marzo de 1989, julio de 1991 y nosotros en febrero de 1996. Nuestra resección se trataba de un tumor grande de 11x8x5 cm, con histología compatible con un lipoma maduro, con fibrosis sin encontrar ninguna atipia.

En noviembre de 1999 acude por nueva recidiva, palpando una masa importante a tensión en zona inguino-escrotal izquierda. Realizamos una ecografía y TAC abdomino-pelviano que nos confirma una gran masa de tipo lipomatoso inguinoescrotal de unos $14 \mathrm{~cm}$ de diámetro (Fig. 1), no visualizando adenopatías retroperitoneales ni pélvicas. Practicamos una amplia incisión inguinal hasta cerca del escroto con resección fácil de una gran masa encapsulada, a diferencia del lipoma resecado anteriormente. Buen curso postoperatorio de sólo 24 horas. El tumor era de 1,4 $\mathrm{Kg}$ de peso, bien circunscrito, amarillento, en áreas de patrón nodular, de coloración gris-blanquecina y amarillentas con zonas necrohemorrágicas (Fig. 2). La histología (Fig. 3) a X400 se puede observar un lipoblasto típico en el centro de la imagen, con adipositos maduros y las zonas de esclerosis del liposarcoma. Hasta aquí el liposarcoma es bien diferenciado con áreas de patrón esclerosante. Sin embargo en el siguiente corte

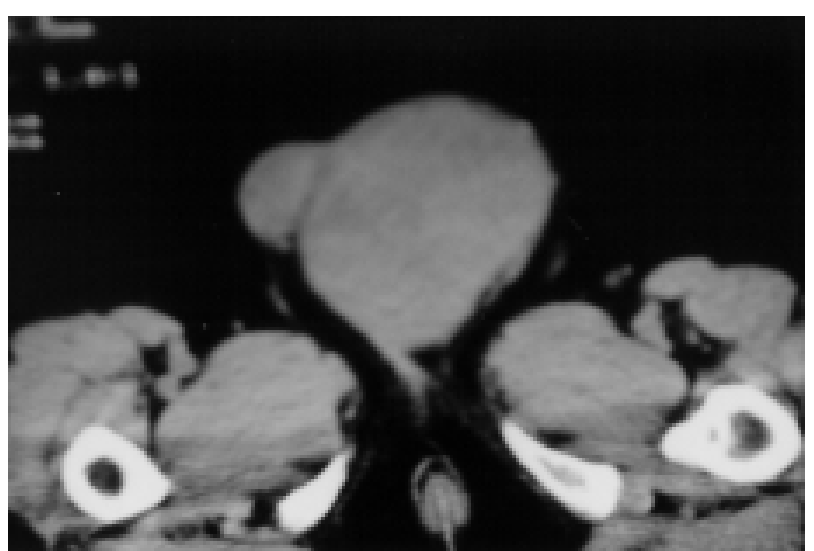

FIGURA 1. TAC. Masa homogénea inguino escrotal de $14 \mathrm{~cm}$.

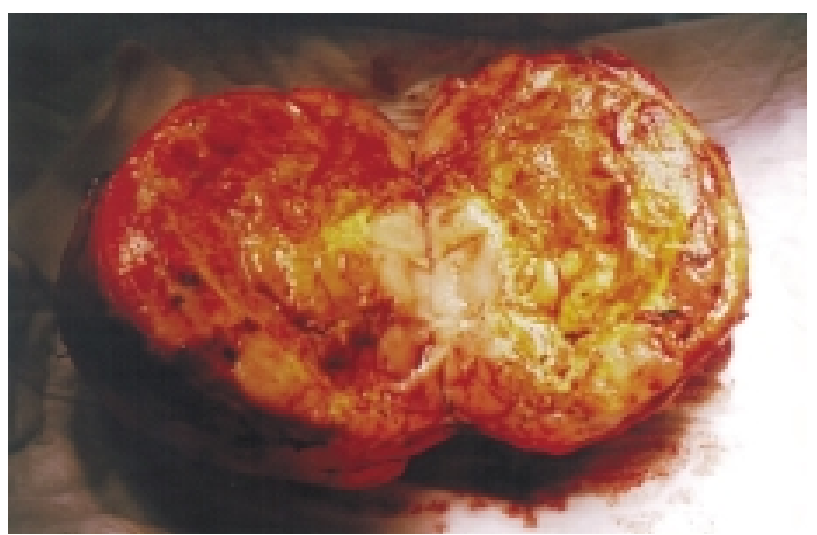

FIGURA 2. Tumor encapsulado, amarillento, con áreas de patrón nodular y un peso de $1,4 \mathrm{~kg}$.

(Fig. 4) X40 vemos el cambio que hay del liposarcoma bien diferenciado de tipo esclerosante a la zona dediferenciada de alto grado. La inmunohistoquímica en estas zonas dediferenciadas muestra positividad de las células tumorales para desmina y vimentina y negatividad para la proteína S-100 y la CAM 5,2. El diagnóstico es de liposarcoma dediferenciado. A los 6 meses el paciente presentó nueva recidiva local, que requirió resección más agresiva y poliquimioterapia.

\section{DISCUSIÓN}

Las neoplasias del cordón espermático tienen el mismo aspecto morfológico que los tumores de los tejidos blandos, siendo los más frecuentes los de estirpe benigna, los lipomas (45\%). Los sarcomas representan el 31\%, salvo los rabdomiosarcomas que es la neoplasia más frecuente en las dos primeras décadas de la vida, siendo histoló- 


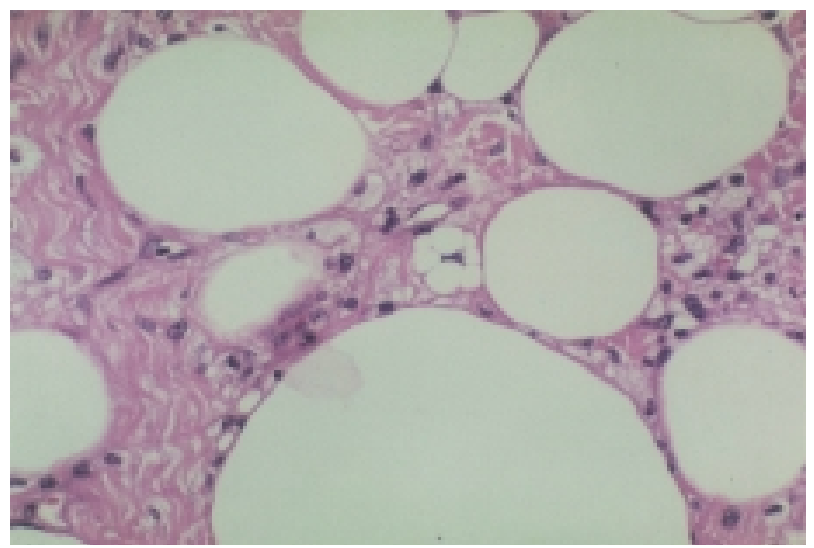

FIGURA 3. En esta figura se puede observar un lipoblasto tipico en el centro de la imagen, con adipositos maduros y las zonas de esclerosis del liposarcoma. Hasta aqui el liposarcoma es bien diferenciado con áreas de patrón esclerosante.

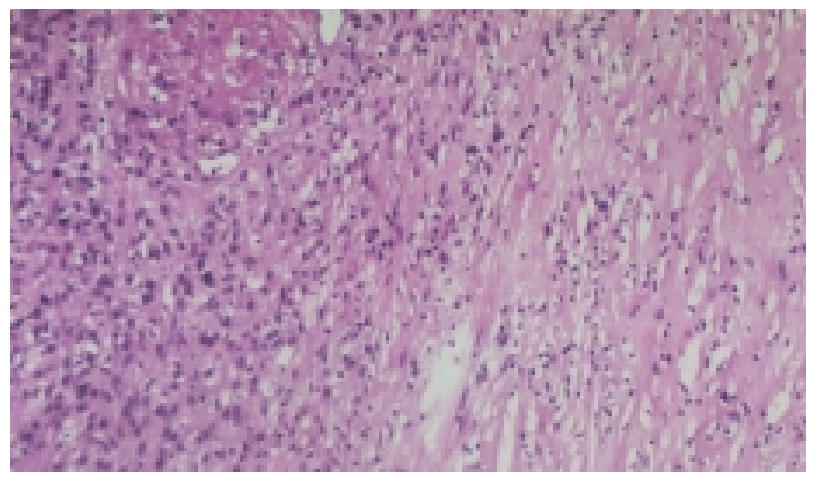

FIGURA 4. Vemos el cambio que hay del liposarcoma bien diferenciado de tipo esclerosante a la zona dediferenciada de alto grado.

gicamente el tipo más frecuente embrionario ${ }^{3}$. En el adulto el liposarcoma, el leiomiosarcoma y el fibrosarcoma son los otros tumores malignos del cordón espermático. Los liposarcomas son tumores mesenquimales malignos que se pueden desarrollar donde quiera que exista tejido adiposo, siendo su localización fundamental el retroperitoneo. Para algunos autores estos tumores serían el resultado de una transformación maligna de un lipoma pre-existente, debido a los diferentes patrones histológicos de diferenciación que existen en la masa tumoral de los liposarcomas de cordón espermático ${ }^{4,5}$.

No obstante otros autores no parecen estar de acuerdo con esta teoría, ya que si provienen de los lipomas pre-existentes, debido a su frecuencia la incidencia de los liposarcomas tendría que ser mayor ${ }^{4,6}$.
La clínica es la de una lesión de tipo lipomatoso grande de 1,5 a $23 \mathrm{~cm}$ (media $5,5 \mathrm{~cm}$ ), masa inguino-escrotal indolora o con sensación de compresión o disconfort, se presenta en adultos más frecuente a partir de 40 años. La evolución de su crecimiento varía según los autores (entre semanas a más de 10 años), desde lento a muy acelerado como en nuestro caso. El diagnóstico preoperatorio se realizará mediante la exploración física, junto con la ecografía, el TAC y la RNM que nos orienta identificando un tejido adiposo en el interior de la masa, nos delimitara la posible afectación inguinal o la existencia de adenopatías pelvianas o retroperitoneales ${ }^{7,8}$. El diagnóstico diferencial se realizará con todas las tumoraciones intraescrotales como primeramente el lipoma, hidrocele, hematocele, hernia inguinal, quiste del cordón y epididimario, epididimitis, etc.

Nuestro enfermo tras 3 resecciones de un lipoma de cordón espermático, presentó un liposarcoma dediferenciado que creemos se trata de una transformación neoplásica. Henricks revisa 155 casos de liposarcomas dediferenciados encontrando anatómicamente esta localización de los mismos: 106 retroperitoneales, 32 tejido blando, y en extremidades siendo el siguiente más numeroso con 13 casos la localización de cordónescrotal $^{10}$. El liposarcoma dediferenciado es un liposarcoma bien diferenciado con áreas de sarcoma de alto grado, áreas de aspecto no lipomatoso de proliferación fusocelular y epitelioide, de crecimiento en sábana. En las zonas de sarcoma de alto grado presenta positividad para desmina (marcador de músculo) y vimentina (marcador de tumor mesenquimal) ${ }^{10,11}$.

El tratamiento de este tipo de tumor es la orquiectomía radical por vía inguinal alta, siendo la quimioterapia y la radioterapia de ayuda, incierta. Estos tumores requieren una estrecha vigilancia debido a la frecuente recidiva a los 5 años: local $(19,4 \%)$ y de metástasis hematógenas $(11,1 \%)$ y de nódulos pelvianos $(5,5 \%)^{2}$.

\section{REFERENCIAS}

1. SÁNCHEZ MERINO JM, PARRA MUNTANER L, GARCÍA ALONSO J.: Tumores paratesticulares. Acción Médica. Madrid, 1997; 7.

2. BALLO MT, ZAGARS GK, PISTERS PW et al.: Spermatic cord sarcoma: outcome, patterns of failure and management. $J$ Urol 2001; 166: 1306-1310. 
3. PEYRI E, NUBIOLA P, HIDALGO F et al.: Lipoma de cordón espermático. Actas Urol Esp 1998; 22 (3): 238.

4. BECCIA DS, KRANE RS, OLSON LA.: Clinical management of nontesticular intraescrotal tumors. J Urol 1976; 116: 476.

5. SOLER JL, ZULUAGA A, HIDALGO MR y cols: Liposarcoma de cordón espermático: aportación de un nuevo caso y revisión de la literatura. Actas Urol Esp 1999; 23 (5): 447-454.

6. DOMÍNGUEZ F, RIERA JR, JUNCO $\mathrm{P}$ y cols.: Liposarcoma bien diferenciado de cordón espermático con desdiferenciación. Arch Esp Urol 1994; 47 (7): 717-720.

7. CALAHORRA FJ, PÉREZ C, RAMOS y cols.: Liposarcoma paratesticular del cordón espermático. Actas Urol Esp 1990; 14 (3): 202-204.

8. SCHWARTS SL, SWIERZEWSKY SJ, SONDAK VK et al.: Liposarcoma of the spermatic cord: report of 6 cases and review of the literature. $J$ Urol 1995; 153 (1): 154-157.
9. EVANS HL.: Liposarcomas and atypical lipomatous tumors: a study of 66 cases followed for a minimum of 10 years. Surg Pathol 1988; 1: 41-54.

10. HENRICKS WH, CHU YC, GOLDBLUM JR et al.: Dedifferentiated liposarcoma. A clinicopathological analysis of 155 cases with a proposal for an expanded definition of dedifferentiaton. Am J Surg Pathol 1997; 21 (3): 271-281.

11. CHAN YF, YUEN MY, LI MK.: Recurrent dedifferentiated liposarcoma of the spermatic cord simulating malignant fibrous histiocytoma: and ultrastructural study. Pathology 1987; 19: 99-102.

Dr. E. Peyrí Rey

C/ Valencia, $247-1^{\circ} 1^{\text {a }}$

08007 Barcelona

(Trabajo recibido el 28 noviembre 2001) 